\title{
Stationary nonlinear waves at the surface of a thin liquid layer under inverted gravitation conditions
}

\author{
G.V. Kolmakov, E.V. Lebedeva, A.A. Levchenko, \\ L.P. Mezhov-Deglin, A.B. Trusov, and V.B. Shikin \\ Institute of Solid State Physics RAS, Chernogolovka, Moscow region, 142432, Russia \\ E-mail: german@issp.ac.ru
}

Received May 21, 2003, revised July 28, 2003

\begin{abstract}
Instability of the flat surface of a thin liquid layer wetting a solid substrate under inverted gravitation conditions is discovered. The development of this instability leads to formation of a new stationary nonuniform liquid surface state. It looks like a solitary hill with characteristics sensitive to the liquid film parameters, particularly to the layer thickness at which the instability begins to develop. By application of a variational approach the mechanical stability of such a hill (droplet) in the one-dimensional approximation is proved. A variational picture of the shape evolution for a cylindrical charged droplet in an external electric field is constructed, too. The results obtained are compared with an experiment on liquid hydrogen droplets [A.A. Levchenko, G.V. Kolmakov, L.P. Mezhov-Deglin, M.G. Mikhailov, and A.B. Trusov, Low Temp. Phys. 25, 242 (1999)]. The theory developed is in good agreement with the results of experiments.
\end{abstract}

PACS: 68.15.+e, 05.45.Yv, 67.70.+n

\section{Introduction}

A charged or neutral liquid layer is an example of a two-dimensional (2D) system that can exhibit a mechanical instability under inverted gravitation conditions, i.e., under conditions when the layer is suspended at the horizontal surface of a solid substrate. It is known that a liquid layer suspended on a solid substrate under inverted gravitation conditions is absolutely unstable if the van der Waals forces are neglected [1]. In the presence of the attractive van der Waals forces between liquid and substrate, there is a finite interval of layer thicknesses for which a mechanical equilibrium is possible, and a sufficiently thin layer covers the substrate uniformly. At thicknesses larger than some critical value, the van der Waals forces cannot keep a liquid layer in a flat state and the instability develops [2].

The mechanical instability of the flat free surface of a neutral liquid layer of a thickness larger than the critical value develops at small wave vectors [2], which is similar to the instability of the charged surface of a thin liquid layer in an external pressing electric field [3-5]. This situation is quite different from the known instability phenomena of charged surfaces of a thick liquid helium layer [6-11], because in this case the instability develops at a finite wave number of the order of the inverse capillary wave length. The scenario of development of the long-wave instability of thin layer surfaces was not clear till now.

The main goal of this paper is to study the nonuniform phenomena that occur on neutral and charged surfaces of a thin liquid layer. We show that the instability leads to the formation of a stationary, nonlinear, solitary wave of surface deformations. The wave amplitude increases with increasing liquid layer thickness or with increasing stretching electric field above some critical values. This behavior is quite different from that of a bulk liquid surface, where the final jump of the deformation wave amplitude takes place at a critical point.

The computed evolution of the shape of the reconstructed surface is compared with the data obtained in earlier experiments [12] on neutral and charged liquid hydrogen layers.

The solution to the problem of stationary surface shapes of thick liquid layers and droplets placed at the surface of a solid substrate was proposed many years ago in Frenkel's papers [13]. In that consideration the van der Waals forces between liquid and substrate 
were neglected, and their interaction was taken into account by fixing the contact angle between the liquid and the solid (the wetting angle). Such a formulation of the problem excludes, by definition, the possibility of considering a transition from the flat to the nonuniform liquid surface shape: in Frenkel's treatment the layer is always in a nonuniform state. As we shall see, the shape of the nonuniform liquid film surface tends to be that of a droplet, as found in [13], only for a sufficiently large volume of the liquid layer.

To simplify the presentation below we propose to introduce a new term - «reconstruction». Reconstruction implies the transition from the flat to the new mechanically stable nonuniform liquid surface state.

The structure of this paper is as follows. Chapter 2 contains the general mechanical equilibrium equation (11) for a liquid film under inverted gravitation conditions, with comments related to the instability origin and corresponding effective energy definition (24).

The one-dimensional version (22) of Eq. (11) is suitable for analytical description of the reconstruction details. We obtain the explicit solution (27), (28) of the one-dimensional reconstruction problem. In contrast to the bulk situation $[6,7,9]$ this solution is not unique. It contains the arbitrary parameter $C$ which should be physically reasonable. One possibility for defining this parameter could correspond to a soliton-like representation of the solution (27). It corresponds to the requirement $T \rightarrow \infty$, where the period $T$ is given by Eq. (28). But this condition is in contradiction with the equation of normalization (14). The second possible picture - the periodic reconstruction - is not valid, too. This follows from the properties of the second variation for the functional $\mathcal{E}$ defined by Eq. (24). Finally, the main conclusion of Chapter 2 is the proof of mechanically stable reconstruction of the inverted liquid film in the form of an individual droplet which is sensitive to the boundary conditions.

The one-dimensional speculations from Chapter 2 enable us to believe that the main characteristics of the reconstruction phenomena remain the same in the $2 \mathrm{D}$ case (certainly, this assumption cannot be proved). The variational picture for an individual radial droplet in the neutral and charged states is constructed (see Chapter 3 ). The results obtained are shown to be in agreement with the currently available experimental data. The corresponding fitting is presented in Chapter 3 , too.

\section{Reconstruction of a thin layer of neutral liquid}

\subsection{Instability of the flat surface of a liquid layer}

We consider first the stability problem for a neutral liquid layer covering a flat horizontal surface of a solid substrate under inverted gravitation conditions (i.e., when the gravitational force is opposite to the attractive van der Waals force between the liquid and substrate). A schematic picture of the system under study is shown in Fig. 1. We introduce the following frame of reference. The $(x, y)$ plane coincides with the substrate surface. The $z$ axis is directed downward, parallel to the gravity force. The free liquid surface is described by the equation $z=\zeta(\mathbf{r})$, where $\mathbf{r}$ is the two-dimensional vector within the $(x, y)$ plane.

The equilibrium shape of the free surface of the liquid layer is governed by three forces: the gravitation force, the Laplace capillary force, and the van der Waals force, which retains the liquid at the substrate. Under inverted gravitation conditions the pressure at the free surface is given by the following expression:

$$
P=-\frac{\alpha \Delta \zeta}{\left[1+(\Delta \zeta)^{2}\right]^{3 / 2}}-\rho g \zeta-P_{w}(\zeta) .
$$

Here $\alpha$ is the surface tension, $\rho$ is the density of the liquid, $g$ is the free fall acceleration, and

$$
P_{w}(\zeta)=\frac{f}{\zeta^{3}}\left(1+\frac{\zeta}{d_{w}}\right)^{-1}
$$

is the pressure of the van der Waals forces ( $f$ is the van der Waals constant and $d_{w}$ is some characteristic width).

Expression (1) contains two terms conventional for the capillary problem (the Laplace and the gravitational pressures, see, e.g., [14]). Besides we take into account the van der Waals ( $\mathrm{vdW}$ ) forces between a liquid layer and a solid substrate (the last term $P_{w}(\zeta)$ of Eq. (1)). The interpolated form (2) for the van der Waals pressure was proposed in [15]. This expression describes two dependencies as limiting cases:

$$
P_{w}(\zeta)=f / \zeta^{3} \quad\left(\text { at } \zeta<<d_{w}\right)
$$

and

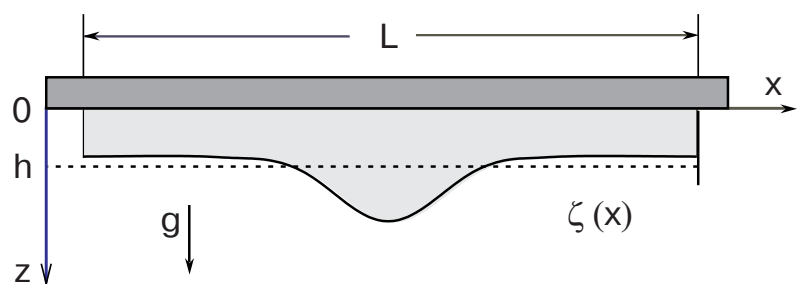

Fig. 1. Schematic view of a reconstructed liquid layer suspended at a substrate. The liquid layer covers a region of the surface of a substrate between vertical walls. $L$ is the distance between the walls, and $h$ is the mean thickness of layer. The gravitational force is directed downward, along the $z$ axis. The equation describing the free surface is $z=\zeta(x)$. 


$$
P_{w}(\zeta)=f d_{w} / \zeta^{4} \quad\left(\text { at } \zeta>>d_{w}\right) .
$$

The asymptotic expression (3) is applicable in the situations of «thin» films, where the retarding effects in the definition of the vdW forces can be neglected, and expression (4) is valid for «thick» films, for which the retarding effects play a significant role. The measurement of the constants $f$ and $d_{w}$ is a special problem. It is possible to use some original techniques [15], or, in the case of superfluid helium, to determine these constants from measurements of the third-sound propagation [16]. For hydrogen films the values of $f$ and $d_{w}$ are not yet known.

Generally speaking, the definition of the local vdW pressure (like in Eq. (1)) is correct for a flat uniform surface only. It can be used, for example, for estimations of an equilibrium liquid film thickness (see Ref. 17). In the nonuniform case the vdW force has an integral form [18,19]. The local approximation used in Eq. (1) is acceptable if

$$
d<<,
$$

where $\lambda$ is the typical nonuniform scale along the liquid surface. For the reconstruction problem under consideration the condition (5) is fulfilled.

If the free surface is flat, and the layer thickness is a constant, $\zeta=h$, then the Laplace term in Eq. (1) turns to zero, and the pressure at the surface is

$$
P=-\rho g h-P_{w}(h) .
$$

One can introduce the effective gravitation acceleration at the liquid surface $g_{\text {eff }}=\rho^{-1}(\partial P / \partial h)$, which controls the net force acting on the surface. The pressure $P$ has an extremum at the thickness $h_{*}$,

$$
\partial P /\left.\partial h\right|_{h=h_{*}}=0, \text { or }\left.\left(-\rho g-\frac{\partial P_{w}(h)}{\partial h}\right)\right|_{h=h_{*}}=0,
$$

where the derivative $\partial P / \partial h$ changes sign. So, with increasing layer thickness from $h<h_{*}$ to $h>h_{*}$, the effective gravitational acceleration $g_{\text {eff }}$ changes sign from positive to negative. This change of the direction of the effective gravitational accelerational implies that the flat state of the free surface should be unstable for thicknesses $h>h_{*}$, and that small disturbances of the flat surface should grow with time.

In the limiting cases of large or of small thicknesses of the film the critical thickness $h_{*}$ is defined as follows:

$$
\begin{gathered}
h_{*}=\left(4 f d_{w} / \rho g\right)^{1 / 5} \quad\left(h_{*}>>d_{w}\right), \\
h_{*}=(3 f / \rho g)^{1 / 4} \quad\left(h_{*}<<d_{w}\right) .
\end{gathered}
$$

Note that a solution of Eq. (6) always exists because the inequality

$$
\frac{\partial P_{w}(h)}{\partial h}<0
$$

holds for any values of the constants $f$ and $d_{w}$.

In the opposite geometry, when the liquid layer covers the upper horizontal surface of the substrate (the gravitational and van der Waals forces have the same direction), the sign of the effective gravitational force is positive for all thicknesses $h$. This is in agreement with the fact that the flat free layer surface is always stable under «normal» gravitation conditions.

As is shown in the next Section, the instability of flat surface at large $h$ leads to reconstruction, which consists in the creation of a stationary, nonlinear wave of deformations of the free surface.

\subsection{Equilibrium shape of the reconstructed liquid surface}

In order to describe peculiarities of the reconstruction at the surface of a liquid layer wetting a horizontal flat substrate, we determine the equilibrium shape of the free surface of the layer taking into account the gravitational, capillary, and van der Waals forces. Generally speaking such a problem reduces to solution of the equilibrium equation

$$
P(\zeta)=\text { const }
$$

where $P(\zeta)$ is defined by Eqs. (1), (2). Equation (10) reflects the fact that in a steady state the pressure at the free surface should be constant over the entire surface. This gives

$$
\frac{\alpha \Delta \zeta}{\left[1+(\Delta \zeta)^{2}\right]^{3 / 2}}+\rho g \zeta+P_{w}(\zeta)+p=0 .
$$

We denote the constant in the right-hand side of Eq. (10) as $p$. The value of the constant $p$ should depend on the shape of the liquid surface and should be determined from «the normalization condition»

$$
\int d^{2} \mathbf{r} \zeta(\mathbf{r})=V,
$$

where $V$ is the total volume of the liquid in the layer, and the integration is over the area of the substrate covered by liquid.

As is shown below, taking the boundary conditions (wetting the substrate by the liquid) into account is of fundamental importance for consideration of instability and reconstruction phenomena. We discuss here a model case in which the layer is restricted in the horizontal direction by vertical walls (see Fig. 1). The effective wetting angle (the angle of slope of the liquid surface at the point of contact between the liquid and the solid surfaces) we denote as $\theta$. The solu- 
tion to the set of equations (11), (12) with the following boundary conditions at the vertical walls,

$$
|\Delta \zeta|=\tan \theta,
$$

will uniquely determine the stationary shape of the liquid surface.

In order to describe the main qualitative features of the reconstruction phenomena we restrict our consideration in this Section to the model case where a one-dimensional (1D) wave of deformation appears at the surface. In this case the function $\zeta$ depends only on one horizontal coordinate, $x$.

The normalization condition (12) in the one-dimensional case reads

$$
\int d x \zeta(x)=S,
$$

where $S$ is the «two-dimensional volume» (area) of the liquid layer.

We suppose, also, that the angle of slope of the free surface with respect to the horizontal plane is small, $\left|\zeta^{\prime}\right|<<1$. As is shown below, this condition holds to a high accuracy when $h<10^{3} h_{*}$, which is true for typical conditions of experiment. In this case one can expand Eq. (11) in the gradient $\zeta^{\prime}$; as a first approximation this equation will read

$$
\alpha \zeta^{\prime \prime}+\rho g \zeta+P_{w}(\zeta)+p=0 .
$$

Note that the deformation of the surface is not assumed to be small in the general case, so we keep the term $P_{w}(\zeta)$ in this equation.

One can show that the development of the instability of the flat surface at the initial stage of the reconstruction, i.e., if

$$
\left(h-h_{*}\right) / h_{*}<<1 \quad\left(h>h_{*}\right),
$$

does not depend on the model behavior (Eq. (4) or Eq. (3)) assumed for the vdW forces. It is shown below that if the inequality (16) holds, then the stationary nonuniform deviation of the reconstructed surface from the initial flat state is small with respect to $h_{*}$. In this case the surface shape can be calculated using a perturbation approach. For this purpose it is convenient to present the deformation of the surface as

$$
\zeta(x)=h_{*}+\xi(x),
$$

where

$$
\frac{|\xi(x)|}{h_{*}} \ll<1 .
$$

By using the representation (17) the equation (15) can be expanded in a series in the small parameter (18). In this way the deviation $\xi(x)$ should be represented as a series

$$
\xi(x)=\xi^{(1)}(x)+\xi^{(2)}(x)+\ldots
$$

The term $\xi^{(1)}(x)$ is a small function of the first order, the term $\xi^{(2)}(x)$ is a small function of the second order, etc. Equation (15) reads as a linear approximation

$$
\alpha\left(\xi^{(1)}\right)^{\prime \prime}+p^{(1)}=0 .
$$

Here $p^{(1)}$ is a first-order correction to the $p$ constant. The second (quadratic) approximation reads

$$
\alpha\left(\xi^{(2)}\right)^{\prime \prime}+\frac{1}{2}\left(\frac{\partial^{2} P_{w}}{\partial h^{2}}\right)_{h_{*}}\left(\xi^{(1)}\right)^{2}+p^{(2)}=0 .
$$

The higher-order equations can be written down in straightforward way. The values of $p^{(1)}, p^{(2)}$ should be found from the normalization condition (14).

It can be seen that the expression $P_{w}$ given by Eq. (2) is dropped from the linearized Eq. (20) due to condition (6), and the derivative of $P_{w}$ (taken at $h=h_{*}$ ) is a factor in the quadratic term of the second-order equation (21), only. This means that the choice of the explicit functional dependence of the $\mathrm{vdW}$ pressure $P_{w}$ on the layer thichness $P_{w}=P_{w}(h)$ from model (3) or from model (4) is not of importance for analyses of the reconstruction transition at $h$ close to $h_{*}$. The derivative $\partial^{2} P_{w} /\left.\partial h^{2}\right|_{h=h_{*}}$ (which can be evaluated easily in both models) controls only the «nonlinearity level» in the system at given $h$.

As we show in this paper, in the opposite case $h>h_{*}$ the main part of liquid in the layer should be accumulated in a droplet of macroscopic dimension formed at the substrate surface. The thickness of the $\mathrm{vdW}$ liquid layer $\zeta_{\min }$ wetting the substrate outside the droplet should remain small. Moreover, this thickness is $\zeta_{\min }<h_{*}$ and it decreases further with increasing total volume $V$ of the liquid. For very large (macroscopic) $V$ the thickness $\zeta_{\min }$ of the vdW wetting layer should become comparable to or even less than $d_{w}$, and in this case the model (3) is more appropriate (see the corresponding estimations in Section 2.3).

Keeping in mind that the goal of this paper is not only to analyze the peculiarities of the reconstruction picture itself but also to compare the results of calculations with the experimental data obtained in [12] for a macroscopic droplet, we will not restrict ourselves to the expansion provided by Eqs. (20), (21) but will consider the more general equation (15). Unfortunately, integration of the Eq. (15) in analytical form with the general expression for $P_{w}(\zeta)$ from (2) is a complicated problem. But this integraton can be done easily if $P_{w}(\zeta)$ is taken in a power-like form (3) or (4). In this paper we restrict our consideration to the limiting case (3) for $P_{w}(\zeta)$. It should be taken into account that the results obtained are valid quantitatively in the 
macroscopic case $h>h_{*}$ (large $V$ ), only. But the qualitative picture of the reconstruction does not depend on the model assumed for the vdW pressure.

Under the above-stated assumptions Eq. (15) reads

$$
\alpha \zeta^{\prime \prime}+\rho g \zeta+\frac{f}{\zeta^{3}}+p=0
$$

In the rest of this chapter the solutions of Eq. (22) determined under the additional constraint (14) are analyzed.

It is useful for the next consideration to note here that Eq. (11) with $P_{w}(\zeta)$ from (3) can be derived from the following variational principle: the total energy of the liquid at equilibrium must reach a minimum, which is found under the additional restriction of Eq. (12), i.e.,

$$
\delta\left[\mathcal{E}-p \int d^{2} \mathbf{r} \zeta(\mathbf{r})\right]=0 .
$$

The total energy of the liquid, which includes contributions from the surface tension, the gravitational force, and the van der Waals force, is

$$
\mathcal{E}=\int d^{2} \mathbf{r}\left[\alpha \sqrt{1+(\Delta \zeta)^{2}}-\frac{1}{2} \rho g \zeta+\frac{f}{2 \zeta^{2}}\right] .
$$

The normalization condition (12) is taken into account in Eq. (23) using the Lagrange undetermined multiplier method, the pressure $p$ at surface of liquid playing the role of that undetermined multiplier.

The first integral of the one-dimensional equation (22) is

$$
\frac{1}{2} \zeta^{2}+U(\zeta)=C
$$

where $U(\zeta)=\left(\rho g \zeta^{4}+2 p \zeta^{3}-f\right) / 2 \alpha \zeta^{2}$.

To analyze the above set of equations, the following mechanical analogy is useful. Equation (25) can be considered as the energy conservation law for an effective point-like particle with mass $m=1$ moving in an external potential, $U(\zeta)$. The variable $x$ plays the role of time, and $\zeta$ is the coordinate of the particle. The first term in Eq. (25) is the kinetic energy of the particle, and $C$ is the total energy.

Figure 2 shows the evolution of the effective potential $U(\zeta)$ with increasing layer thickness. Figure 2,a illustrates the case of small layer thicknesses $h<h_{*}$, where $h_{*}$ is defined by Eq. (6). The point of the maximum $\zeta=h$ on the $U(\zeta)$ curve corresponds to a stable flat surface of the thin liquid layer. The «velocity» of the particle at this point is $\zeta^{\prime}=0$, and $C=U(h)$.

With increasing $h$ up to the critical value $h_{*}$ the curve $U(\zeta)$ transforms into a curve with an inflection point at $h=h_{*}$ (see Fig. 2,b). If $h>h_{*}$, the dip on the curve develops again (Fig. 2,c). The point of the minimum of the function $U(\zeta)$ at some $\zeta>h_{*}$ corresponds to an unstable flat state of the liquid surface. Formation of the possible stationary surface deformation wave is described by periodic motion of the particle in the potential well around the minimum in the region where $U(\zeta)-C<0$.

The coordinates of the turning points that restrict the motion of the particle can be found from the equation $U(\zeta)-C=0$. In the general case this equation has four different solutions, $\zeta=z_{i}, i=1, \ldots, 4$, which are the roots of the polynomial

$$
\rho g \zeta^{4}+2 p \zeta^{3}-2 \alpha C \zeta^{2}-f .
$$

We will enumerate these roots in the following order: $z_{4}<z_{3}<z_{2}<z_{1}$.

Now it is reasonable to remind ourselves that we are trying to find a possible nonuniform solutions of the problem (22). In the case of an infinite film area $-\infty<x<+\infty$ the nonuniform shape of the surface $\zeta(x)$ can be periodic, or, under some special conditions, a soliton-like one. Therefore we should obtain the periodical solutions of Eq. (22) and investigate their properties, first.
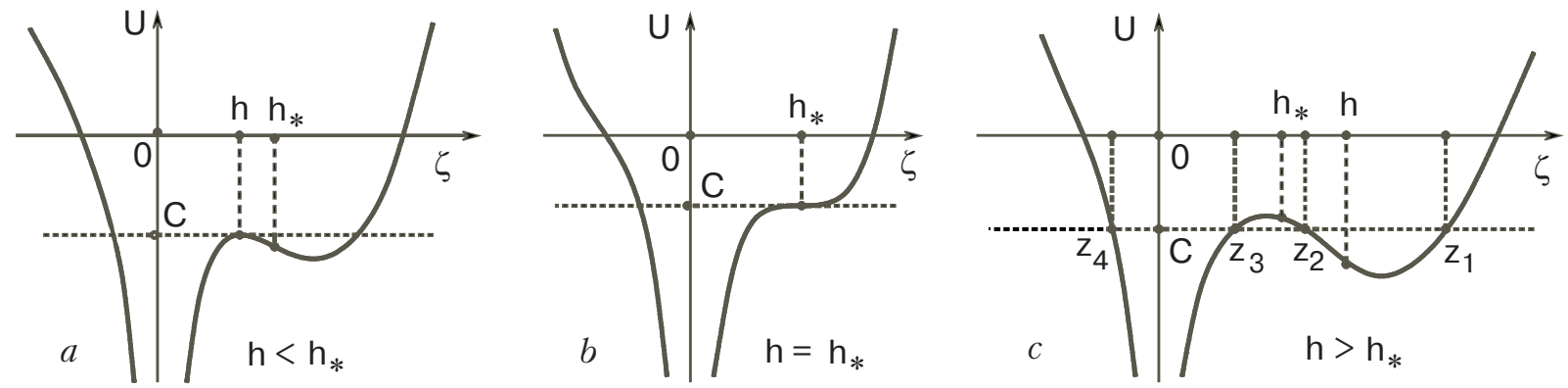

Fig. 2. Evolution of the effective potential $U(\zeta)$ with increasing mean layer thickness $h . a-$ small thickness, $h<h_{*}$; $b$ - the thickness of layer is equal to the critical value $h_{*} ; c-$ the thickness $h>h_{*}$. The points $z_{i}, i=1, \ldots, 4$ correspond to solutions of the equation $U(z)=C$. 
The general periodic solution to Eq. (25) in the region $z_{2}<\zeta<z_{1}$ can be written in integral form as follows:

$$
\int_{z_{2}}^{\zeta} \frac{d \zeta}{\sqrt{2[C-U(\zeta)]}}=x-x_{0} .
$$

The arbitrary parameter $x_{0}$ in the right-hand side of Eq. (26) appears due to the invariance of the initial equation (11) with respect to translations in the horizontal plane.

The integral in the left-hand side of Eq. (26) can be expressed via the elliptic integrals of the first and third kinds, $F(\mu, r)$ and $\Pi(\mu, n, r)$, as follows:

$$
\begin{gathered}
\frac{2}{\sqrt{\left(z_{1}-z_{3}\right)\left(z_{2}-z_{4}\right)}}\left[\left(z_{2}-z_{3}\right) \Pi\left(\lambda, \frac{z_{1}-z_{2}}{z_{1}-z_{3}}, r\right)+\right. \\
\left.+z_{3} F(\mu, r)\right]=\frac{x-x_{0}}{a},
\end{gathered}
$$

where

$$
\begin{gathered}
\lambda=\arcsin \sqrt{\frac{\left(z_{1}-z_{3}\right)\left(\zeta-z_{2}\right)}{\left(z_{1}-z_{3}\right)\left(\zeta-z_{3}\right)}}, \\
r=\sqrt{\frac{\left(z_{1}-z_{2}\right)\left(z_{3}-z_{4}\right)}{\left(z_{1}-z_{3}\right)\left(z_{2}-z_{4}\right)}},
\end{gathered}
$$

and $a=(\alpha / \rho g)^{1 / 2}$ is the capillary length.

Equation (27) defines in implicit form the reconstructed shape of the free surface of the liquid layer covering the solid substrate under inverted gravitation conditions. The period $T$ of the surface deformation wave defined by Eq. (27) is equal to

$$
\begin{gathered}
T=\frac{4 a}{\sqrt{\left(z_{1}-z_{3}\right)\left(z_{2}-z_{4}\right)}} \times \\
\times\left[\left(z_{2}-z_{3}\right) \Pi\left(\frac{\pi}{2}, \frac{z_{1}-z_{2}}{z_{1}-z_{3}}, r\right)+z_{3} F\left(\frac{\pi}{2}, r\right)\right] .
\end{gathered}
$$

We should mention that the roots $z_{i}$ depend on two free parameters, i.e., on the pressure $p$ at the surface and on the constant of integration $C$ (in addition to the dependence on the constants characterizing the properties of the liquid and its interaction with the substrate). The solution defined by Eq. (27) should obey the normalization condition (14), too. This condition provides an additional relation between parameters $p$ and $C$. Thus one can conclude that the function $\zeta$ depends on one free parameter, in addition to the dependence on the $x$ coordinate. This parameter can be chosen arbitrarily. In the subsequent analysis we suppose that the constant $C$ plays the role of this parame- ter. The period $T$ of the stationary wave depends on this parameter, as well.

One possibility of avoiding the problem nonuniqueness of the parameter $C$ could be the realization of a soliton-like solution for $\zeta(x)$. Following the conventional recommendation (see, e.g., the problem of steady-state wave propagation at the free liquid surface in Ref. 20) we have to use for this purpose the additional requirement

$$
T \rightarrow \infty .
$$

However, this requirement turns out to be incompatible with the normalization condition (14), because in this case the integral

$$
\int d x(\zeta(x)-h)
$$

diverges. Therefore the conventional soliton-like solution is not valid.

Now, turning back to the general periodic picture of reconstruction, we have only one possibility for resolving the problem of nonuniqueness of the parameter $C$. Here it is necessary to use stability arguments. Using the results obtained below (see paragraph 2.3) as a verification, we can show that the deformation $\zeta(x)$ with the maximal period $T$ is the most favorable energetically. For the case of unbounded geometry $-\infty<x<+\infty$ this means that the period of the structure on the reconstructed surface should tend to infinity. Therefore energetically stable periodic reconstruction of the unbounded thin helium film is impossible, too.

Nevertheless, we can bring the factor of stability into the formulation of the problem artificially, by considering a film of limited area. Such a scenario corresponds to the experimental situation. So, let us assume that

$$
-L / 2 \leq x \leq L / 2
$$

and, in addition, that the following boundary conditions are fulfilled:

$$
\left.\zeta^{\prime}\right|_{ \pm L / 2}=0 .
$$

The simplest boundary conditions (32) with the contact angle at the walls $\theta=0$ are necessary to save the basic instability indicator $h_{*}$ in the form (6).

After reformulation (31), (32) the dependence of the function $\zeta(x)$ defined by Eqs. (27), (28) on the parameter $C$ changes its character. Under the new boundary conditions $C$ can take only a definite discrete set of values. Now our objective is to demonstrate that the most energetically preferable solution can be found among the solutions corresponding to these values. 


\subsection{Reconstruction of the free surface of a thin layer in restricted geometry}

Following the speculations above, we have to investigate the solutions (27) under the boundary conditions (32).

The values of the parameter $C$ at which the boundary conditions (32) are satisfied can be found from the requirement that the ratio $L / T$ is equal to some natural number $N$. The number $N$ coincides with the number of maxima of the function $\zeta(x)$ in the region $-L / 2<x<L / 2$. So, such a solution describes the formation of $N$ humps, or «droplets», at the liquid surface. These droplets are connected between each other by the liquid layer covering the substrate.

Detailed numerical analysis of the stability problem for the solution with $N$ droplets at the surface shows that only the solution with $N=1$ can be realized in experiment; see below.

First, the energy of the liquid layer with $N$ droplets at the surface ( $\ll N$ droplet configuration») is estimated numerically. The results of estimations show that the configuration with $N=1$ corresponds to a minimum of the energy, while the energy of configurations with $N>1$ is higher. Figure 3 shows an example of two possible stationary profiles of the reconstructed surface with $N=1$ (curve 1) and $N=2$ (curve 2) calculated for case $h=1.78 h_{*}$. The horizontal coordinate $x$ is expressed in units of the capillary length $a$, and the vertical coordinate is expressed in units of the critical thickness $h_{*}$. The distance between the vertical walls is $L=13.3 a$. The line with zero vertical coordinate is the substrate surface. The horizontal dashed line corresponds to the flat layer of thickness $h=h_{*}$. The estimated energy of the configurations is expressed in energy units $E_{0}=(\alpha \rho g)^{1 / 2} h^{2} / 4$. The energy of the configuration with $N=1$ (the profile has one maximum) is equal to $E_{1}=-3.80 E_{0}$, while the energy of the configuration with two maxima is equal to $E_{2}=-2.86 E_{0}$. So, the configuration with $N=1$ is energetically preferable and should be realized in experiment.

Now we study the stability of the solution with a single maximum against small perturbations of the surface. For this purpose the sign of the second variation of the energy $\delta^{(2)} E$ at small perturbations of the shape of the liquid surface is determined. For a stable solution the second variation is positive definite (a solution should correspond to a minimum of the total energy). The variation $\delta^{(2)} E$ is represented by the following functional, which is quadratic in the small perturbation $\delta \zeta(x)$ of the steady-state surface profile $\zeta(x)$ :

$$
\delta^{(2)} E=\int_{-L / 2}^{L / 2} d x \delta \zeta(x) \hat{\Lambda} \delta \zeta(x),
$$

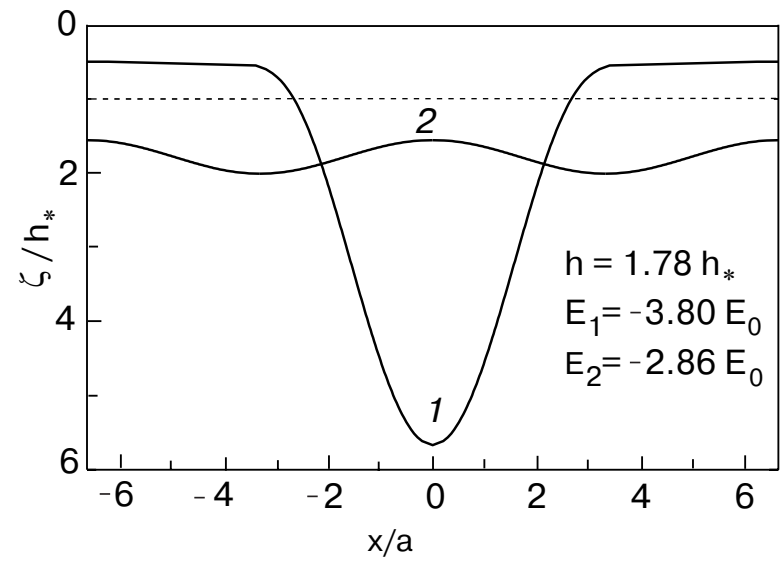

Fig. 3. Results of a calculations of the stationary shape of the reconstructed layer with a single droplet (curve 1) and with two droplets (curve 2) formed at the surface. The $x$ coordinate is normalized to the capillary length $a$, and the layer thickness is normalized to the critical thickness $h_{*}$. The distance between the vertical walls is equal to $L=13.3 a$. The mean thickness of the layer is $h=1.78 h_{*}$. The dashed line corresponds to the flat surface of a layer with $h=h_{*}$. The estimated energy of the single- and two-droplet solutions are $E_{1}=-3.80 E_{0}$, and $E_{2}=-2.86 E_{0}$, where $E_{0}=\sqrt{\alpha \rho g} h^{2} / 4$.

where

$$
\hat{\Lambda}=-\frac{\alpha}{2} \frac{\partial^{2}}{\partial x^{2}}-\frac{\rho g}{2}+\frac{3 f}{\zeta^{4}(x)}
$$

is a linear differential operator. The positive definiteness of the second variation of energy is equivalent to the fact that the operator $\hat{\Lambda}$ has only positive eigenvalues $\lambda_{n}>0$. The problem on finding the spectrum $\lambda_{n}$ of the operator $\hat{\Lambda}$ can be mapped onto the well-known problem of the energy spectrum of a quantum particle with mass $m=1 / \alpha$ moving in the potential well $U=-\rho g / 2+3 f / \zeta^{4}(x)$. We study the stability problem for the case when the perturbation does not affect the wetting conditions at the walls, so the boundary conditions for the «wave function» are $\delta \zeta^{\prime}(x)=0$ at $x= \pm L / 2$. It is clear from this consideration that the perturbation that corresponds to the lowest eigenvalue of the «Hamiltonian» $\hat{\Lambda}$ (i.e., to the «ground state level») is the most dangerous for destroying the stability of the steady-state surface shape.

The low-energy part of the spectrum of the operator $\hat{\Lambda}$ is found numerically, by direct diagonalization of the matrix corresponding to the operator $\hat{\Lambda}$. The matrix elements $\Lambda_{m n}$ are calculated in an orthogonal normalized basis presented by a set of symmetric $(g)$ and antisymmetric $(u)$ functions

$$
\sqrt{2 / L} \cos \left(q_{n}^{(g)} x\right), \quad \sqrt{2 / L} \sin \left(q_{n}^{(u)} x\right),
$$


where $q_{n}^{(g)}=2 \pi(n+1) / L$,

and $q_{n}^{(u)}=\pi(2 n+1) / L, n=0,1,2, \ldots$

The function $\zeta(x)$ is symmetric with respect to inversion of the $x$ coordinate, $\zeta(-x)=\zeta(x)$. This means that the matrix is diagonal with respect to the index of the perturbation parity, i.e., only the matrix elements with two $u$ or two $g$ indices are nonzero. This enables examination of the stability problem against symmetric and antisymmetric perturbations separately. The matrix element calculated for two basis functions of the same parity corresponding to excitation levels $n$ and $m$ is

$$
\Lambda_{n m}=\left[\frac{\alpha}{2}-\frac{\rho g}{2}\right] \delta_{m n}+3 f\left(\frac{1}{\zeta^{4}(x)}\right)_{n m},
$$

where $\delta_{n m}$ is the Kroneker delta, and the index $u$ or $g$ is omitted. The elements $\left(1 / \zeta^{4}\right)_{n m}$ are calculated numerically for the lowest 10 harmonics, $n, m \leq 10$. Numerical estimations show that taking higher harmonics $(n, m>10)$ into account does not change noticeably the character of the lower part of the spectrum of the operator $\hat{\Lambda}$. We study the case where $L<15 a$ and $h<10 h_{*}$. Analysis show that all eigenvalues are positive, $\lambda_{n}>0$. This means that small perturbations of the reconstructed surface with $N=1$ increase the total energy, and, hence, the shape of the steady state of the surface is stable.

Such a direct stability analysis can not be done successfully by numerical reasoning if the mean thickness of the liquid layer $h>10 h_{*}$ or the dimension $L>15 a$, due to losses of accuracy in the numerical calculations of the values of roots $z_{i}$.

Meanwhile, at high $h$ one can use the following qualitative considerations to demonstrate the fact that the solution with one droplet at the reconstructed surface is energetically preferable, and only this configuration can be formed [21]. In the general situation, the profile of the steady state of the reconstructed surface corresponds to the formation of $N$ droplets at the surface, which are connected by the liquid layer covering the substrate. In the limiting case where the volume of liquid is macroscopically large, the thickness of such a layer is small with respect to the height of the droplet. The main part of the liquid is accumulated in droplets, and the volume of liquid contained in the layer covering the substrate between the droplets is negligibly small. As a first approximation the shape of each droplet can be described by the 1D Frenkel solution (see Ref. 13)

$$
\zeta(x)=\frac{A_{0}}{2}(1+\cos x / a),
$$

where the height of the droplet is

$$
A_{0}=S / \pi a,
$$

and $S$ is the «two-dimensional volume» (area) of the droplet. The energy of a single droplet whose shape is described by Eq. (33) can be calculated directly. This gives

$$
E_{1}(S)=-\frac{\alpha}{2 \pi a^{3}} S^{2} .
$$

The total energy of the liquid can be estimated as a sum of energies of each «isolated» droplet. If total volume $S$ of liquid is distributed among $N$ identical droplets, than the energy of such a configuration can be estimated as follows:

$$
E=N E_{1}(S / N)=-\frac{\alpha}{2 \pi a^{3}} \frac{S^{2}}{N} .
$$

So, the energy of the liquid is increased if the liquid is redistributed from one droplet into a number of droplets. In the macroscopic case $\left(h \gg h_{*}\right)$ the solution with one droplet formed inside a cell is also preferable from energy considerations.

From the results obtained it follows that the consideration of the reconstruction process can be restricted to the case where a single droplet is formed at the surface. Below we present results of numerical computations of the evolution of the shape of the reconstructed layer with one droplet formed at the surface with increasing mean layer thickness $h$.

Figure 4 shows the shape of the reconstructed surface obtained by numerical integration of Eq. (22) in cases where the mean liquid layer thickness is equal to $h=1.5 h_{*}$ (curve 1 ), $2.2 h_{*}$ (curve 2 ), and $7 h_{*}$ (curve 3 ). The $x$ coordinate at the graph is expressed in capillary

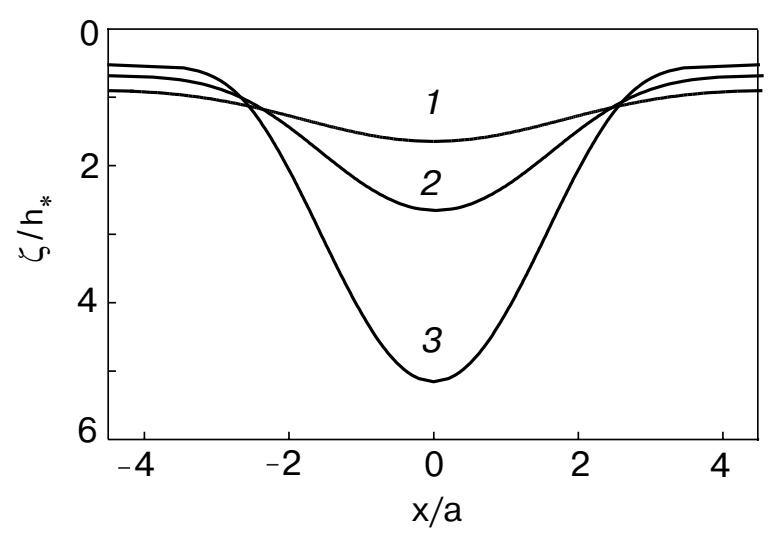

Fig. 4. Evolution of the shape of the reconstructed liquid layer with a single droplet at the center with increasing mean thickness $h$. Curve 1: $h=1.5 h_{*}$, curve $2: h=2.2 h_{*}$, curve $3: h=7 h_{*}$. The thickness of the layer is expressed in units of the critical thickness. The distance between the vertical walls is $L=9 a$. 
length units $a$, and the $z$ coordinate is expressed in the critical thickness units $h_{*}$. The distance $L$ between the vertical walls is equal to $9 a$. The $z$ coordinate of the substrate surface is equal to zero.

It can be seen from Fig. 4 that the amplitude of the wave of surface deformation increases gradually with increasing $h$ above $h_{*}$. The maximum of the deformation is situated at the center of the cell. The wave acquires a droplet-like shape at sufficiently large $h$. The characteristic horizontal size of the droplet is of the order of a few capillary lengths, and it depends weakly on the mean thickness $h$. The thickness $\zeta_{\min }=\zeta( \pm L / 2)$ of the liquid layer covering the substrate outside the droplet is less than the critical thickness $h_{*}$, and it decreases with increasing mean thickness $h$. In case $h>h_{*}$ the main part of the liquid is concentrated in the droplet, and the volume of liquid in the thin layer wetting the substrate outside the droplet is much smaller than the total liquid volume.

As was shown in [21]

$$
\left(\frac{\zeta_{\min }}{h_{*}}\right)^{3} \simeq \frac{h_{*}}{3 \zeta_{\max }}, \quad \zeta_{\max } \simeq A_{0}+\zeta_{\min } .
$$

Here $A_{0}$ is given from Eq. (34). The combination of Eq. (37) with the requirement $\zeta_{\min }<d_{w}$ leads to estimations of the critical $S_{\min }$, which is necessary for simplification (3). So, it should be

$$
S>S_{\min }, \text { where } S_{\min }=\pi a h_{*}^{4} / 3 d_{w}^{3} .
$$

Estimate (38) confirms the propositions made in the preceding qualitative analysis.

Figure 5 shows the dependence of the droplet height $A=\zeta(0)-\zeta(L / 2)$ on the parameter $\delta=\left(h-h_{*}\right) / h_{*}$ (the overcriticality parameter). For comparison of our results with the results of macroscopic considerations [13], the droplet height $A$ is normalized to the height $A_{0}$ given by Eq. (34) for Frenkel's droplet, i.e., on the height of the droplet formed under inverted gravitation conditions from a liquid of the same volume $S=L h$ and calculated in case where the angle of wetting of the substrate (not the walls) by the liquid is $\theta=0$. In these calculations the horizontal dimension of the cell is $L=9 a$, which is the same as in Fig. 4 .

It follows from Fig. 5 that at high thickness $h>>h_{*}$ (i.e., at high overcriticality level, $\delta>>1$ ) the height of the droplet formed at the center of the cell is close to the height of Frenkel's droplet of the same volume. The difference between the heights of the «van der Waals droplet» and Frenkel's droplet is about $10 \%$ at $\delta \approx 6$ and decreases with increasing $\delta$. Thus, at a layer thickness one order of magnitude higher than the critical thickness $h_{*}$ the shape of the droplet formed

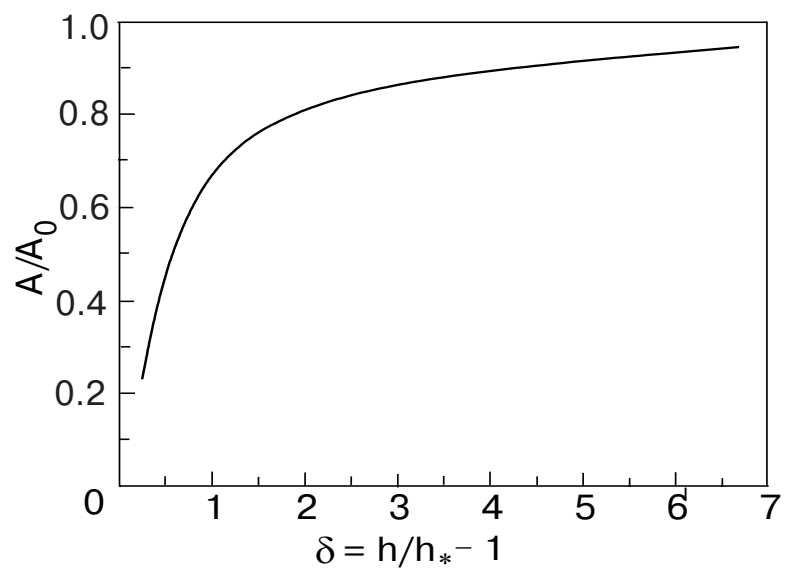

Fig. 5. Dependence of the amplitude of the wave of surface deformation on the overcriticality parameter $\delta=h / h_{*}-1$. Amplitudes are measured in units of $A_{0}$, where $A_{0}=S / 4 \pi a$ is the amplitude of Frenkel's droplet, and $S$ is the total area of one-dimensional layer.

under inverted gravitation conditions can be described to rather good accuracy by the «macroscopic» solution (33). The effective angle of wetting of the substrate by liquid at the edges of the macroscopic droplet is

$$
\theta_{\text {eff }}=0 .
$$

The characteristic horizontal dimension of the droplet is of the order of capillary length at $\delta>1$. So, if the overcriticality level is not very small, and the dimensions of the cell are much larger than $a$, then the boundary conditions should have a weak effect on the equilibrium shape of the droplet.

At $h \sim h_{*}$ the dependence of the droplet height $A=A(h)$ differs significantly from that obtained in the macroscopic approximations, and at $h=h_{*}$ (at the overcriticality $\delta=0$ ) the height of the droplet tends to zero. At thicknesses smaller than the critical thickness the flat liquid surface is stable, and the layer is in the nonreconstructed state (no deformation wave appears at the surface). Details of the behavior of the surface shape at $h$ close to $h_{*}$ depend on the value of the wetting angle $\theta$. The corresponding analysis will be published elsewhere.

Let us consider in addition to (38) the limitations for applicability of the results obtained. The main assumption in the treatments presented is the small angle approximation, $\left|\zeta^{\prime}(x)\right|<<1$. The characteristic length scales in the horizontal and vertical directions differ drastically. The horizontal size of the deformation wave is of the order of the capillary length. For liquid hydrogen it is equal to $a \approx 0.2 \mathrm{~cm}$. The characteristic scale of thickness is presented by the critical thickness $h_{*}$, and in the case of a hydrogen film covering a dielectric substrate it is $h_{*} \approx 10^{-4} \mathrm{~cm}$. The condition at which our treatment fails can be written 
as $A / a \sim 1$, or, in equivalent form, $h \sim a^{2} / L$. One can conclude from these estimations that the results of our considerations are valid (from the side of large thickness) if $\delta<a / h_{*} \sim 10^{3}$, or $h<10^{-1} \mathrm{~cm}$, for $L \sim a$. From the side of small thickness the applicability of the results is restricted to the possibility of considering the liquid as a continuous medium, i.e., $h>10^{-6} \mathrm{~cm}$. So, there is a macroscopically wide interval of layer thickness where the results obtained are valid.

The problem of the stationary shape of a liquid droplet can be solved without the assumption of smallness of the gradient of the surface deformation if the capillary forces are taken into account (see, e.g., [13]). This assumption is of importance for the presentation of the van der Waals pressure as a power law of the layer thickness in Eq. (1). The condition $\left|\zeta^{\prime}(x)\right|<<1$ is of crucial importance for calculations of the electric pressure at the surface of charged liquids, too (see the next Section).

\section{Macroscopic charged droplet formed at a solid substrate}

\subsection{Equipotentially charged surface of a liquid in an electric field}

In this Section the evolution of the shape of a charged droplet with increasing mass of the droplet and external electric field strength is studied. Calculations are carried out for two cases, where a droplet is suspended at a metallic substrate or it is lying on the substrate. In the first case the direction of the stretching electric force acting on the liquid surface is the same as the direction of the gravity force, and in the second case the forces have opposite directions. These situations correspond to conditions of experiments [12] with liquid hydrogen droplets. In the experiments the upper or the lower plate of a horizontally arranged flat capacitor play the role of the substrates.

It is supposed that the charges are localized under the free surface of the liquid and they create a quasi-two-dimensional layer, which totally screens the electric field in the bulk of the liquid (i.e., the electric field and the density of charges in the bulk of the liquid are equal to zero). The electric potential at the liquid surface is maintained equal to some constant value by an external battery, and the total number of positive charges localized under the surface can be varied due to a continuously working source of charges, which is placed in the bulk of the liquid.

A macroscopic situation will be considered here, i.e., the height of the droplet is supposed to be much larger than the critical thickness $h_{*}$. As was pointed out above, a typical value of the critical thickness is of the order of $10^{-4} \mathrm{~cm}$, and it is smaller than droplet heights suitable for optical observations. The influence of the van der Waals forces on the shape of the macroscopic droplet is negligibly small, and one can exclude it from considerations. Meanwhile, the van der Waals forces are comparable with the electric and gravitational forces at the edges of the droplet, where the liquid depth becomes the same order as the thickness of thin liquid layer wetting the substrate, i.e., of the order of the critical thickness. As is shown in the previous Section, the influence of the wetting layer at the substrate surface on the shape of the droplet could be taken into account by using the effective boundary conditions (39) that read $\zeta^{\prime}=0$ at the edges of the droplet. The thin liquid layer wetting the substrate outside the droplet should be considered to have zero thickness $(\zeta=0)$ in the macroscopic consideration used in this Section.

It was shown above that almost all the volume of a neutral liquid covering a substrate is concentrated in a single droplet at the substrate surface. It is natural to assume that this property also holds for a charged liquid, and a single droplet should appear at the equipotentially charged surface of the liquid.

Here we consider a real «two-dimensional» situation in which the shape of the liquid surface depends on two coordinates in the $(x, y)$ plane. We also assume that the droplet formed at the surface has an axially symmetric shape, i.e., the liquid surface is described by an equation $z=\zeta(|\mathbf{r}|)$.

Calculations of the equilibrium shape of the droplet are carried out in two steps. First, the effective energy of the charged droplet formed at the capacitor plate is calculated as a function of the shape of the droplet and of the voltage $U$ applied to the capacitor. After that, the dependence of the height $A$ and the effective radius $R$ of the droplet is found using the variation approach, from the condition that the effective energy acquires a minimal value at the equilibrium state.

Let us consider a droplet suspended at the upper plate of the flat horizontal capacitor (i.e., the droplet under inverted gravitation conditions). The equilibrium shape of the liquid droplet with an equipotentially charged surface corresponds to a minimum of the effective energy (see Ref. 22)

$$
\mathcal{E}=\mathcal{E}_{\text {mech }}-\frac{1}{8 \pi} \int d^{2} \mathbf{r} \int_{\zeta}^{d} d z \mathbf{E}^{2} .
$$

Here $\mathcal{E}_{\text {mech }}$ is the mechanical energy of the liquid (a sum of the surface energy and the energy of the liquid in the gravitational field), the last term in the right-hand side presents the energy of the electric field in the capacitor, $\mathbf{E}$ is the electric field strength in the 
gas phase, and $d$ is the distance between the capacitor plates. The frame of reference is chosen the same as used in the previous Section: the $(x, y)$ plane coincides with the surface of the upper capacitor plate, at which the droplet is suspended, and the $z$ axis is directed along the gravitational force.

The form of the expression for the electric field energy in a capacitor depends on the ratio between the horizontal size of the droplet (its effective radius $R$ ) and the distance $d$ between the capacitor plates. In the calculations presented below we assume that the condition $d / R<<1$ holds.

Also, in order to simplify the calculations it is assumed that the height of the $\operatorname{droplet} A$ is small with respect to the distance $d, A<<d$.

From this it follows that the angle of the slope of the surface with respect to the horizon is given in order of magnitude by a product of two small quantities, $|\nabla \zeta| \sim \zeta / R \sim(\zeta / d) \times(d / R)$, and it is a small quantity of high order. Restrictions for applicability of the results obtained, which follow from these assumptions, will be discussed later.

In order to determine the contribution of the electric field energy to the total effective energy (40) one should calculate the electric potential $\varphi$ inside the capacitor. The electric potential obeys Laplace's equation and the boundary conditions at the upper and lower plates

$$
\Delta \varphi=0,\left.\quad \varphi\right|_{z=\zeta(\mathbf{r})}=0,\left.\quad \varphi\right|_{z=d}=U .
$$

The value of the electric potential at the charged surface of a droplet suspended at the upper plate is assumed to be equal to the value of the potential at the plate. This value is chosen equal to zero. The value of the potential at the lower plate is denoted as $U$.

It is convenient to introduce a new potential $\psi$ defined by the equation $\psi=\varphi+E_{0} z$, where $E_{0}=-U / d$. As follows from Eq. (41), the boundary conditions for the $\psi$ potential are the following:

$$
\left.\psi\right|_{z=\zeta(\mathbf{r})}=E_{0} \zeta,\left.\quad \psi\right|_{z=d}=0 .
$$

In the case under study, $\zeta<<d<<R$, the potential $\psi$ could be calculated using perturbation theory, with the liquid layer thickness $\zeta$ considered as a small function,

$$
\psi=\psi^{(1)}+\psi^{(2)}+\ldots,
$$

where $\psi^{(1)} \sim \zeta, \psi^{(2)} \sim \zeta^{2}$, etc. In order to determine the boundary conditions for the functions $\psi^{(n)}$ one should substitute the expansion (43) into Eq. (42) and equate the terms of the same order. For the first-order terms the conditions read

$$
\left.\psi^{(1)}\right|_{z=d}=0,\left.\quad \psi^{(1)}\right|_{z=0}=E_{0} \zeta .
$$

We assume that the droplet has an axially symmetric shape, i.e., $\zeta=\zeta(r)$, where $r=|\mathbf{r}|$. The solution of the problem of the potential distribution with boundary conditions (44) is known from potential theory. In the region $\zeta \leq z \leq d$, where the electric field is not equal to zero, the solution can be found using a Bessel transformation. It reads

$$
\begin{aligned}
& \psi^{(1)}(r, z)=E_{0} \int_{0}^{\infty} d t J_{0}(r t) F(t) t \frac{\operatorname{sh} t(d-z)}{\operatorname{sh} t d}, \\
& \text { where } F(t)=\int_{0}^{\infty} d r r J_{0}(r t) \zeta(r),
\end{aligned}
$$

and $J_{0}(t)$ is the Bessel function of order zero.

The Bessel transform $F(t)$ of the function $\zeta(r)$ is essentially nonzero if its argument $t \leq 1 / R$. Thus the arguments of the hyperbolic functions in the right-hand side of Eq. (45) are of order $d / R<<1$, and these functions can be expanded in series in their arguments. Below we need the expression for the derivative of the $\psi^{(1)}$ function. Calculations give the following relation

$$
\partial \psi^{(1)} /\left.\partial z\right|_{z=0}=-E_{0} \zeta / d .
$$

Here we use the inverse Bessel transformation in the calculations.

We will calculate the dependence of the electric field energy on the shape of the droplet up to third-order accuracy in the ratio $\zeta / d$. The energy of the electric field in the capacitor is expressed via the $\psi$ potential as

$$
\mathcal{E}_{\mathrm{el}}=\frac{1}{8 \pi} \int d^{2} \mathbf{r} \int_{\zeta}^{d} d z(\nabla \psi)^{2},
$$

where we have neglected an inessential constant in the right-hand side of (47). In the same third-order approximation this expression can be reduced to an integral over the surface of the upper plate as follows:

$$
\mathcal{E}_{\mathrm{el}}=-\frac{E_{0}}{8 \pi} \int d^{2} \mathbf{r}\left(\zeta\left[\frac{\partial \psi^{(1)}}{\partial z}\right]_{z=0}+\zeta^{2}\left[\frac{\partial^{2} \psi^{(1)}}{\partial z^{2}}\right]_{z=0}\right)
$$

It follows from the relation obtained that the energy of the electric field written up to the third-order terms is expressed via the first order term of the $\psi$ potential and via the $\zeta$ function. One can exclude the $\psi^{(1)}$ function from the expression (48) using Eq. (46) and a similar equation for the second derivative of the potential obtained from the integral representation (45). This gives the following dependence of the en- 
ergy of electric field in the capacitor on the droplet shape:

$$
\mathcal{E}_{\mathrm{el}}=\frac{U^{2}}{8 \pi d} \int d^{2} \mathbf{r}\left(\frac{\zeta^{2}}{d^{2}}+\frac{\zeta^{3}}{d^{3}}\right) .
$$

One can see from this expression that the energy of the electric field depends locally on the thickness of the liquid layer $\zeta(r)$. This results from the assumption that the effective radius of the droplet is large with respect to the distance between the capacitor plates.

In calculating the contribution from the surface energy to the mechanical energy of the liquid to the same accuracy, one should take into account only the lowest-order terms in the angle of slope of the surface in the expression for the capillary energy.

The total effective energy of the system (40) calculated in the approximation adopted is given by the following expression:

$$
\begin{gathered}
\mathcal{E}=\frac{\alpha}{2} \int d^{2} \mathbf{r}(\nabla \zeta)^{2}-\frac{1}{2} \rho g \int d^{2} \mathbf{r} \zeta^{2}- \\
-\frac{U^{2}}{8 \pi d} \int d^{2} \mathbf{r}\left(\frac{\zeta^{2}}{d^{2}}+\frac{\zeta^{3}}{d^{3}}\right) .
\end{gathered}
$$

Note that the electric energy in (40) has the opposite sign to the mechanical energy.

\subsection{Equilibrium shape of a charged droplet}

Let us determine the dependence of a shape of a charged droplet on the voltage $U$ applied to the capacitor. A nonlinear equation describing the shape of the droplet can be derived using a variational principle from expression (50) for the effective energy of the system in the same way as was done for Eq. (1). But the solution of the equation obtained in the $2 \mathrm{D}$ situation meets difficulties because of the absence of a first integral of the equation. In this work we determine the height and the radius of the droplet using a variational approach in which the shape of the droplet is approximated by some trial function.

We seek the coordinate dependence of the trial function $\zeta$ in a general axially symmetric form

$$
\zeta(\mathbf{r})=A f\left(\frac{r}{R}\right),
$$

where $A$ is the droplet height and $R$ is the effective radius of the droplet. Parameters $A$ and $R$ are positive variables playing the role of variational parameters in the problem. The function $f(x)$ is a bell-shaped function of the variable $x=r / R$. It is normalized by the conditions $f(0)=1$, and $f(x) \rightarrow 0$ at $x>>1$ (recall that the effective wetting angle at the edges of the droplet is equal to zero).
The dependence of the total energy of the liquid on the variational parameters $A$ and $R$ can be calculated by substituting the function (51) into expression (50). This gives the following expression

$$
\mathcal{E}=\frac{\alpha}{2} c_{0} A^{2}-\left(\frac{1}{2} \rho g+\frac{U^{2}}{8 \pi d^{3}}\right) c_{2} A^{2} R^{2}-\frac{U^{2}}{8 \pi d^{4}} c_{3} A^{3} R^{2},
$$

where the constants $c_{n}$ are defined as follows:

$$
\begin{gathered}
c_{0}=2 \pi \int_{0}^{\infty} d x x f^{\prime 2}(x), \\
c_{n}=2 \pi \int_{0}^{\infty} d x x f^{n}(x) \quad(\text { at } n>0) .
\end{gathered}
$$

The region of integration in (53) is enlarged to infinity due to convergence of the integrals.

The values of the variational parameters in the equilibrium state can be determined from the condition that the effective energy of the liquid acquires a minimal value under the additional normalization condition (12). The normalization condition can be expressed via the variational parameters as

$$
c_{1} A R^{2}=V .
$$

The condition (54) can be taken into account using the Lagrange multipliers method, as was done earlier in calculations of the stationary shape of the neutral liquid surface. Thus the values of the parameters in the equilibrium state can be determined from the equations

$$
\frac{\partial \tilde{\mathcal{E}}}{\partial A}=0, \quad \frac{\partial \tilde{\mathcal{E}}}{\partial R}=0,
$$

where $\tilde{\mathcal{E}}=\mathcal{E}-p A R^{2}$ and $p$ is the Lagrange multiplier.

The solution of Eqs. (55) gives the following dependence of the droplet height $A$ on the voltage $U$ :

$$
A=k_{1} V\left(\frac{U^{2}}{4 \pi d^{3}}+\rho g\right)\left(\alpha-\frac{k_{2} U^{2} V}{4 \pi d^{4}}\right)^{-1} .
$$

The constants in (56) are equal to $k_{1}=c_{2} / 2 c_{0} c_{1}$, $k_{2}=c_{3} / c_{0} c_{1}$.

Note that the function $f(x)$ enters into expressions (54), (56) only as an integrand in the constants $k_{1}, k_{2}$ and $c_{1}$. Thus the choice of the explicit form of the function $f(x)$ has only a weak effect on the calculated values of the droplet sizes.

In order to estimate the values of the constants in (56) we choose the function $f(x)$ in the form 


$$
\begin{gathered}
f(x)=q\left(J_{0}(x)-J_{0}\left(\beta_{1}\right)\right), \quad 0<x<\beta_{1}, \\
f(x)=0, \quad x>\beta_{1} .
\end{gathered}
$$

Here $\beta_{1} \approx 3.83$ is the minimal value of the argument of the Bessel function of the first order, at which the function goes to zero, $J_{1}\left(\beta_{1}\right)=0$, and $q=\left(1-J_{0}\left(\beta_{1}\right)\right)^{-1} \approx$ $\approx 0.71$ is a normalization constant. The explicit form (57) of the $f(x)$ function is chosen from the following considerations. The function (57) is a solution of the equation which describes the liquid surface shape in a linear approximation over the droplet's height. The exact shape of the droplet should be close to that function at a small nonlinearity level in the system, i.e., when the inequalities $|\nabla \zeta|<<1, A / d<<1$ hold.

Values of the constants estimated from definition (53) using the function (57) are $k_{1}=0.0754$, $k_{2}=0.106, c_{1}=18.5$.

The dependence of the effective radius of the droplet on the voltage $U$ can be calculated from Eq. (54) as $R=\left(V / c_{1} A\right)^{1 / 2}$, where $A$ is given by Eq. (56). The diameter of the droplet $D$ (i.e., the diameter of the circle basement of the droplet) can be estimated as $D \approx 7.66 R$.

Let us analyze the result obtained. The droplet height $A$ estimated from (56) grows infinitely when the voltage is increased up to the value

$$
U_{c 2}=\left(\frac{4 \pi \alpha d^{4}}{k_{2} V}\right)^{1 / 2} .
$$

At $U>U_{c 2}$ Eqs. (55) have no solution. At these voltages the effective energy of the droplet as a function of amplitude $A$ decreases with increasing $A$ and, hence, has no minimum. That means that a suspended droplet is absolutely unstable at high voltages. Thus the voltage $U_{c 2}$ given by Eq. (58) can be considered as an estimate for the second critical voltage, at which the liquid droplet loses mechanical stability in the external electric field. At voltages higher than $U_{c 2}$, discharge from the surface accompanied by transfer of liquid from the suspended droplet to the lower plate of the capacitor should take place. Expression (58) should be considered only as an order-of-magnitude estimate because in the case where the amplitude $A$ is comparable with the distance $d$ the theory developed is of a qualitative character.

Dependence (56) can be used to describe the evolution of the shape of the droplet formed from a liquid layer covering the lower plate of the capacitor, with increasing voltage. In this case the stretching electric force, which acts on the equipotentially charged surface of the droplet, is directed oppositely to the gravitational force. To obtain the voltage dependence of the amplitude of a droplet lying on a substrate one should change the sign of the constant $g$ in Eq. (56). This gives the formula

$$
\begin{gathered}
A=0 \quad \text { at } U<U_{c 1}=\sqrt{4 \pi \rho g d^{3}}, \\
A=k_{1} V\left(\frac{U^{2}}{4 \pi d^{3}}-\rho g\right)\left(\alpha-\frac{k_{2} U^{2} V}{4 \pi d^{4}}\right)^{-1} \text { at } U>U_{c 1} .
\end{gathered}
$$

Note that the voltage $U_{c 1}$ coincides with the critical voltage at which the reconstruction of a thin equipotentially charged layer of liquid takes place, see Ref. 3. From the dependence (59) one could see that a droplet is formed at the surface of liquid layer if the «renormalized» gravitation acceleration $g_{\text {eff }}=$ $=g-U^{2} / U_{c 1}^{2}$ changes its sign from positive to negative with increasing voltage $U$. Thus at $U>U_{c 1}$ the surface of the liquid layer condensed on the lower plate of the capacitor could be considered, as a first approximation, to be under «inverted gravitation conditions», where the effective gravitational acceleration is directed oppositely to the real gravitational force. This situation is quite similar to the case of the reconstruction of the thin van der Waals liquid layer considered in the previous Sections. But here the renormalization of the gravitational acceleration that acts on the liquid arises from the electric forces.

As one can see from Eq. (58), the second critical voltage $U_{c 2}$, at which the droplet surface becomes unstable, does not depend on the gravitational acceleration. So, in the «small angle» approximation used the voltages at which the discharge from the surface should take place have the same values in the cases of lying and suspended droplets.

We use the $A(U)$ dependence given by Eq. (56) for treatment the results of experiments [12] with charged hydrogen droplets suspended at a metal substrate. In these experiments the droplet was created by condensation of hydrogen from a gas phase on a cooled upper plate of a horizontally arranged capacitor. This technique allows one to control the layer thickness over a wide range by using precise control of the substrate temperature and of the volume of gas introduced into the experimental cell.

In these experiments the evolution of shape of the suspended charged droplets with increasing voltage applied to the capacitor plates and with increasing volume of the droplet was studied.

Figure 6 shows the changes of the profile of the droplet with increasing voltage $U$, obtained by analisis of snapshots of the droplet. The volume of the droplet was equal to $V=60 \mathrm{~mm}^{3}$ in this experiment. The distance between the capacitor plates was $d=3 \mathrm{~mm}$. The experimental data in Figs. 7 and 8 are reproduced from 


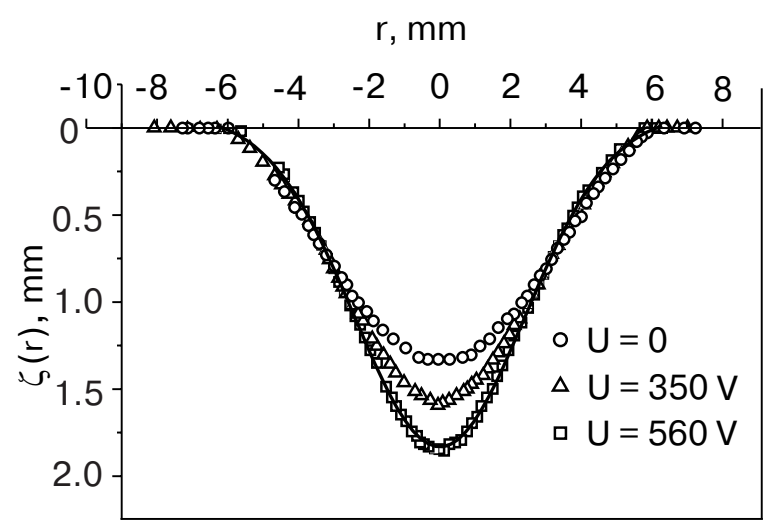

Fig. 6. Profiles of the droplet obtained by scanning snapshots of the suspended charged hydrogen droplet at different voltages $U$ applied to the capacitor.

Figs. 8 and 9 of Ref. 12. Figure 7 shows the dependence of the droplet height $A$ on the voltage $U$ obtained from plots in Fig. 6. The points represent the results of measurements, and the solid curve the dependence (56). Note that the voltage at which the discharge of the surface was observed is equal to $U_{c 2} \approx 800 \mathrm{~V}$. Figure 8 demonstrates the evolution of the dependence of the height of the droplet on the droplet volume with increasing voltage $U$. The solid circles show the dependence of the amplitude $A$ on the volume $V$ measured at zero voltage $U=0$. The squares show the same dependence measured at $U=630 \mathrm{~V}$. The dashed lines correspond to the theoretical dependencies (56) calculated at the given voltages.

In the experiments the instability of a droplet at high voltages, accompanied by a discharge from the droplet surface, was also observed. The values of the second critical voltage $U_{c 2}$ measured in the experiments were approximately three times smaller than the values estimated from Eq. (58). Such discrepancy

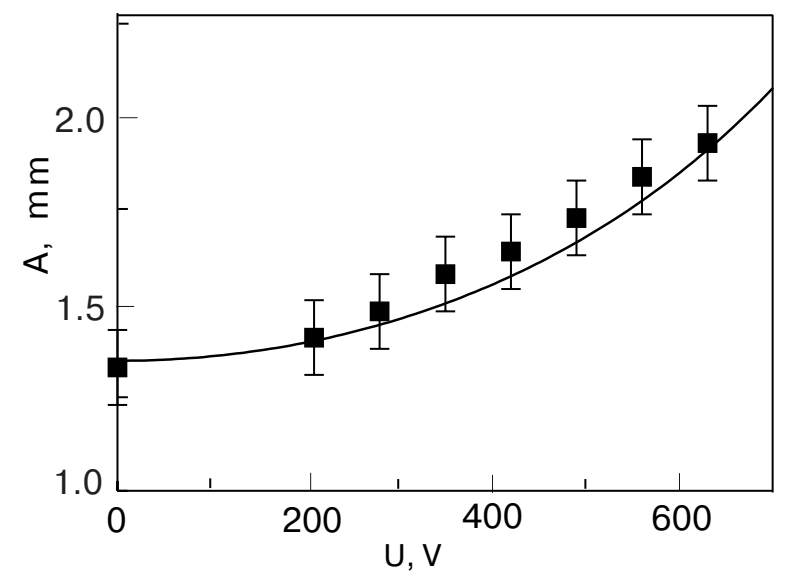

Fig. 7. Dependence of the height of the suspended droplet on the voltage applied to the capacitor. The volume of the droplet is equal to $V=60 \mathrm{~mm}^{3}$. Points - experiment, line - theoretical dependence (56).

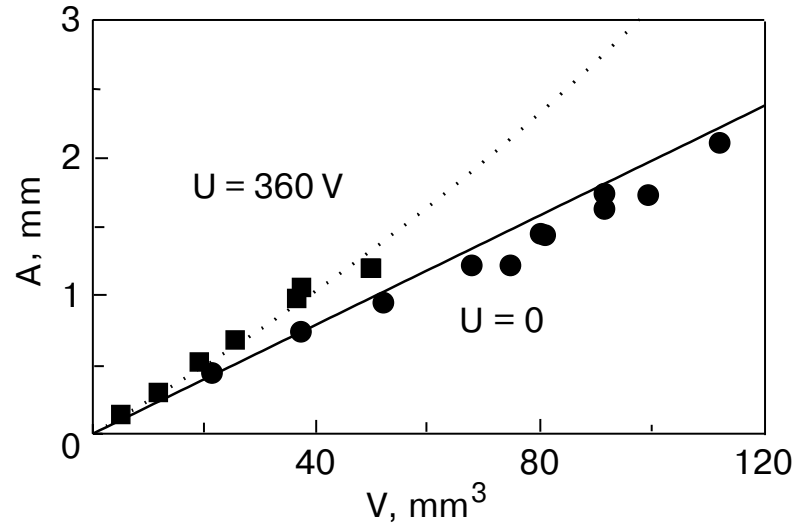

Fig. 8. Dependence of the droplet height on the volume of a neutral droplet, $U=0$ (circles) and of a charged droplet, $U=360 \mathrm{~V}$ (squares). Points - experiment, lines the dependence (56).

can be attributed to the fact that the theory developed is of a qualitative character at voltages close to $U_{c 2}$. The observed character of the development of the instability is different from that assumed in the calculations. Observation showed that the discharge was accompanied by the creation of a «geyser» from the surface, similar to that observed in experiments with the charged surfaces of bulk liquid hydrogen and helium $[12,23]$. The diameter of the geyser stream was much less than the capillary length $a$ of the liquid, so the electric field has high gradients near the stream surface, and the discharge process can not be considered in the framework of the small-gradient approximation used in this paper. The charged surface becomes unsteady, and the appearance of electric current in the system should be taken into account, too. The mechanism of the geyser stream formation could be similar to that studied in the paper [24] for singularity formation at the surface of liquid metal at high electric field.

\section{Conclusions}

It follows from our considerations that the flat surface of a thin neutral liquid film suspended at a solid substrate becomes unstable under inverted gravitation conditions if the thickness of the layer exceeds a critical value $h_{*}$. Development of the instability leads to the formation of a stationary wave of deformation of the liquid surface, i.e., to reconstruction of the surface. In contrast with the periodic reconstruction of the charged surface of a thick layer, the stable shape of the wave corresponds to the formation of a single hump at the center of a cell. The amplitude of the deformation wave increases with increasing the mean thickness $h$ of a layer. If the mean thickness of the liquid layer exceeds significantly the critical thickness, 
$h>10 h_{*}$, the shape of the droplet can be described with good accuracy by Frenkel's macroscopic model, in which the effective boundary conditions of full wetting should be used.

The van der Waals forces are responsible for stabilization of the thin liquid background - a film wetting the substrate outside the droplet. The presence of such a film is important for transitions between states with several humps at the substrate to the state with a single hump.

The application of an electric field to the equipotential charged surface of liquid layer leads, in a first approximation, to renormalization of the gravitational acceleration (to an increase of the effective gravitational acceleration if the droplet is suspended at the metallic plate, and to a decrease of the effective gravitational acceleration if the droplet lies at the plate). The results of the theoretical considerations are in good agreement with experimental observations.

\section{Acknowledgments}

This work is supported in part by INTAS grant 2001-0618, RFBR grant 01-02-97037 and RFBR grant 03-02-16865-a. G.K. also thanks the Science Support Foundation (Russia) for support. We thank M. Strzhemechny and J. Klier for the helpful discussions and useful remarks.

1. D. Book and E. Ott, Phys. Fluids 17, 676 (1974).

2. V.B. Shikin and E.V. Lebedeva, J. Low Temp. Phys. 119, 469 (2000).

3. D.M. Chernikova, Fiz. Nizk. Temp. 2, 1374 (1976) [Sov. J. Low Temp. Phys. 2, 669 (1976)].

4. A.A. Levchenko, E. Teske, G.V. Kolmakov, P. Leiderer, L.P. Mezhov-Deglin, and V.B. Shikin, JETP Lett. 65, 572 (1997)

5. G.V. Kolmakov and E.V. Lebedeva, JETP 88, 24 (1999).
6. L.P. Gor'kov and D.M. Chernikova, Pis'ma v ZhETF 18, 119 (1973).

7. L.P. Gor'kov and D.M. Chernikova, Dokl. Akad. Nauk USSR 228, 829 (1976).

8. V.B. Shikin and P. Leiderer, Fiz. Nizk. Temp. 23, 624 (1997) [Low Temp. Phys. 23, 468 (1997)].

9. V.I. Melnikov and S.M. Meshkov, Zh. Eksp. Theor. Phys. 82, 191 (1982).

10. P. Leiderer and M. Wanner, Phys. Lett. A73, 189 (1979).

11. V.B. Shikin and Yu.P. Monarkha, 2D Charged Systems in Helium, Nauka, Moscow (1989).

12. A.A. Levchenko, G.V. Kolmakov, L.P. Mezhov-Deglin, M.G. Mikhailov, and A.B. Trusov, Fiz. Nizk. Temp. 25, 333 (1999) [Low Temp. Phys. 25, 242 (1999)].

13. Ya.I. Frenkel, Zh. Eksp. Teor. Phys. 18, 659 (1948); Ya.B. Aron and Ya.I. Frenkel, Zh. Eksp. Teor. Phys. 19, 807 (1949).

14. L.D. Landau and E.M. Lifshitz, Hydrodynamics, Nauka, Moscow (1986).

15. E. Sabisky and E. Anderson, Phys. Rev. A7, 720 (1973).

16. S. Putterman, Superfluid Hydrodynamics, North Holland Publish Company, New York (1974).

17. H. Etz, W. Gomber, and P. Leiderer, Phys. Rev. Lett. 53, 2567 (1984).

18. Yu.P. Monarkha, Fiz. Nizk. Temp. 18, 210 (1992) [Low Temp. Phys. 18, 145 (1992)].

19. Yu. Monarkha, U. Albrecht, K. Kono, and P. Leiderer, Phys. Rev. B47, 13812 (1993).

20. M.A. Lavrent'ev and B.V. Shabat, Methods of Theory of Functions of Complex Variable, Nauka, Moscow (1987).

21. V.B. Shikin, JETP Lett. 72, 260 (2000).

22. L.D. Landau and E.M. Lifshits, Electrodynamics of Continuous Media, Pergamon (1975).

23. A.P. Volodin, M.S. Khaikin, and V.S. Edelman, Pis'ma v ZhETF 26, 707 (1977).

24. N.M. Zubarev, JETP Lett. 73, 544 (2001). 